\title{
Metodologías dinámicas en el área musical. Propuesta didáctica: los indios
}

DOI: $10.46932 / \mathrm{sfjdv2n1-035}$

Received in: November 1st, 2020

Accepted in: December 30th, 2020

\author{
Jose María Rabal Alonso \\ Profesor ISEN Centro Universitario \\ Institution: ISEN \\ Calle Campus Universitario, 12, 30100 Murcia \\ E-mail: josemaria.rabal@um.es \\ Andrea Ganga Cano \\ Graduada Educación Infantil \\ Institution: RG Formación \\ Calle Rosalía de Castro, 44, 30107 Murcia \\ Marta Bermejo Zárate \\ Graduada Educación Infantil \\ Institution: RG Formación \\ Calle Rosalía de Castro, 44, 30107 Murcia
}

\section{RESUMEN}

Este trabajo de investigación consiste en una secuencia de enseñanza dirigida a alumnos y alumnas de un aula de 4 años. La temática principal de este serán los indios, pero incluidos dentro del área de la música. El objetivo más importante que queremos conseguir es potenciar el conocimiento de esta materia a través de un tema que puede ser globalizador y una asignatura que será de gran interés para el alumnado y la puesta en práctica de las actividades incitará a los pequeños a participar. Con ayuda de la música, el alumnado estará motivado e incentivaremos la creatividad y la imaginación.

No obstante, es un tema interesante con el que vamos a trabajar diversas partes del currículo del segundo ciclo de Educación Infantil. La secuencia estará compuesta por 4 sesiones o actividades que expondremos detalladamente y, para cada una de ellas, estableceremos contenidos y objetivos. Además, explicitaremos los recursos y la metodología y crearemos dos instrumentos para la posterior evaluación, tanto del alumnado como de la acción docente.

Palabras clave: organización escolar, didáctica, música, emociones, aprendizaje.

\section{JUSTIFICACIÓN}

La música debe ser introducida en Educación Infantil debido a la importancia que representa en su desarrollo intelectual, auditivo, sensorial, del habla y motriz.

La música es un elemento fundamental en esta primera etapa del sistema educativo. El niño empieza a expresarse de otra manera y es capaz de integrarse activamente en la sociedad, porque la música le ayuda a lograr autonomía en sus actividades habituales, asumir el cuidado de sí mismo y del entorno, y ampliar su mundo de relaciones. Y es que los beneficios de la música de los niños son incontables. 
Por esto hemos elegido la temática de los indios para el desarrollo de esta unidad didáctica musical, teniendo en cuenta siempre el currículo de Educación Infantil, y trabajando de forma interdisciplinar sin aislar la música del resto de materias.

Hemos escogido este tema para trabajar la música en el aula de Educación Infantil al observar el interés de los alumnos por la figura del indio, ya que un niño trajo al aula elementos típicos de esta cultura tras haber realizado un viaje a América con su familia durante las vacaciones de Semana Santa. Además, en el resto de áreas también utilizaremos esta temática para trabajar.

\section{CONTEXTUALIZACIÓN}

El CEIP "Maravilla”, se trata de un centro público de doble línea, por lo que cada nivel educativo está constituido por dos grupos de alumnos y alumnas. Éste está ubicado en Santo Ángel, una pedanía o barriada perteneciente al municipio de Murcia, situada unos 5,3 km al sur del centro de la capital de la Región de Murcia (España), en el área sub-comarcal denominada Cordillera Sur. La mayoría de las familias pertenecen a una clase social media.

Atendiendo a las características del alumnado podemos decir que las dificultades presentadas se resuelven en el aula de N.E.E., con apoyos y en la propia aula de su grupo clase. Hay poco alumnado con problemas extraordinarios de necesidades educativas especiales.

La clase a la que va destinada esta unidad didáctica es el aula de 4 años $\mathrm{A}$, con veinticuatro alumnos, de los cuales catorce son niñas y diez niños. En estaclase hay una niña que presenta N.E.E. por lo que necesita adaptaciones curriculares en algunos casos. Esta niña tiene un diagnóstico inicial de trastorno del espectro autista.

Existen diferentes grupos con ritmos de aprendizaje distintos, pero hay un grupo bastante grande de alumnos y alumnas con un buen desarrollo, y esto hace que los que van más lentos se animen y motiven fijándose en los avances de sus compañeros/as.

En cuanto al aula, podemos decir que está estructurada por rincones de trabajo. Los rincones que hay en el aula se pueden dividir en tres, rincones de trabajo, rincones lúdicos y zona de asamblea, estos son:

- Rincones de trabajo: rincón de lógico-matemáticas, rincón de la biblioteca, rincón de la plástica.

- Rincones lúdicos: rincón del juego simbólico y rincón de construcciones.

- Zona de la asamblea.

Los alumnos y alumnas están agrupados en tres equipos de ocho componentes cada uno. Los 
Tabla 1. Temporalización de las actividades.

\begin{tabular}{|c|c|c|c|}
\hline SESIÓN 1 & SESIÓN 2 & SESIÓN 3 & SESIÓN 4 \\
\hline $09 / 05 / 2019$ & $16 / 05 / 2019$ & 23/05/2019 & $30 / 05 / 2019$ \\
\hline Actividad 1: & Actividad 2: & Actividad 3: & Actividad 4: \\
\hline $\begin{array}{l}\text { "Juego con ritmos: La } \\
\text { tribu comanche" }\end{array}$ & $\begin{array}{l}\text { "Tocamos con } \\
\text { instrumentos una canción } \\
\text { india" }\end{array}$ & $\begin{array}{l}\text { "Elaboramos un palo de } \\
\text { lluvia" }\end{array}$ & $\begin{array}{l}\text { Excursión al monte: } \\
\text { "Bailamos alrededor del } \\
\text { fuego" }\end{array}$ \\
\hline
\end{tabular}

Fuente: Elaboración propia.

\section{OBJETIVOS}

Tabla 2. Objetivos de la Unidad Didáctica.

\section{Objetivos curriculares Objetivos específicos}

Participar en juegos colectivos respetando las reglas Participar en todas las actividades propuestas tanto establecidas y valorar el juego como medio de relación individuales como de forma colectiva, mostrando social y recurso de ocio y tiempo libre. interés hacia

ellas.

Observar y explorar de forma activa su entorno, Observar y explorar el entorno natural cercano al generando interpretaciones sobre algunas situaciones y centro, relacionándolo con el ambiente de la temática. hechos significativos y mostrando interés por su

conocimiento.

Conocer distintos grupos sociales cercanos a su Conocer los indios de América, su cultura, sus hábitos experiencia, algunas de sus características, y sus costumbres, además de los instrumentos típicos y producciones culturales, valores y formas de vida, su uso.

generando actitudes de confianza,

respeto y aprecio.

Iniciarse en la audición musical, reconociendo Interpretar las canciones características de los indios, fragmentos musicales de diversos estilos, y progresar, tanto cantadas como representadas a través de la con confianza, en el desarrollo de sus expresión corporal.

posibilidades artísticas y corporales.

Realizar actividades de representación y expresión Confeccionar instrumentos típicos de los indios artística mediante el empleo de diversas técnicas.

mediante técnicas plásticas con

las que estamos familiarizados.

Fuente: Elaboración propia. 


\section{CONTENIDOS}

Tabla 3. Contenidos de la Unidad Didáctica.

\section{Contenidos curriculares Contenidos específicos}

Los objetos y materias presentes en el medio, sus funciones y Interés por la exploración de objetos presentes en el medio y sus usos cotidianos. Interés por su exploración y actitud de respeto y funciones o usos. Respeto por los objetos propios y ajenos. cuidado hacia

los objetos propios y ajenos.

Acercamiento a las costumbres y señas de identidad asociadas a Conocimiento de las costumbres y hábitos relacionados con la la cultura de los países

donde se habla la lengua extranjera.

Comprensión y representación de poesías, $\quad$ Comprensión y representación de canciones

canciones y textos muy sencillos.

Participación en realizaciones colectivas.

Interés y respeto por las elaboraciones

plásticas propias y de los demás. sencillas relacionadas con la temática.

Exploración de las posibilidades sonoras de la voz, del propio Exploración de las posibilidades sonoras del cuerpo, la voz, cuerpo, de objetos cotidianos y de instrumentos musicales. objetos cotidianos e instrumentos musicales. Uso de dichos Utilización de los sonidos hallados para la interpretación y la creación musical. sonidos para la interpretación canciones, juegos musicales y danzas.

Participación activa y disfrute en el aprendizaje e interpretación Interés e iniciativa por aprender canciones, juegos musicales y de canciones, juegos musicales y danzas, siguiendo distintos danzas propias de los indios de América, siguiendo ritmos y ritmos

y melodías, individualmente o en grupo.

melodías

diferentes, individual o colectivamente.

Participación en actividades de dramatización, danzas, bailes, Participación en actividades musicales que conllevan juego simbólico y otros juegos de expresión corporal. Interés e movimientos con el cuerpo: juegos, representaciones, iniciativa para

participar en representaciones.

dramatizaciones, bailes y

danzas.

Fuente: Elaboración propia.

\section{ACTIVIDADES}

- SESIÓN 1: Actividad 1. "Juego con ritmos: La tribu comanche"

- Duración: Esta sesión tendrá una duración de 60 minutos.

\section{- Desarrollo:}

Se trata de una actividad de motivación para introducir a los niños en el tema de la unidad didáctica, en este caso, "Los indios", y que de esta manera sientan interés por él. Consiste en presentar a los niños la canción de "La tribu comanche" para que, posteriormente, hagan ritmos con ella. Para esto, seguiremos los siguientes pasos:

- Presentación del texto de la canción, donde iremos diciendo cada verso de la canción y los niños irán repitiéndolo.

o Presentación del tono melódico, donde haremos lo mismo que anteriormente, pero usando el tono adecuado.

- Acompañamiento, momento en el que se les enseñará el ritmo de la canción utilizando la 
percusión corporal.

○ Realización de un juego: después de todas estas fases, los alumnos y alumnas deberán saber la letra y el ritmo de la canción, además de encajar correctamente la percusión corporal como les hemos indicado. Sentados en círculo en la asamblea, cantaremos la canción todos juntos al mismo tiempo con el ritmo incluido, y al final la maestra señalará a un alumno para que diga su nombre precedido por la frase "yo soy el indio...”. A continuación, cantaremos la canción de nuevo y, esta vez, el alumno señalado escogerá a otro compañero para que diga su nombre usando la misma estructura, y así sucesivamente hasta que todos hayan participado en el juego.

\section{- Materiales:}

En esta actividad se utilizarán como materiales la PDI, el ordenador y los altavoces.

\section{- Organización:}

Esta sesión se va a llevar a cabo en la zona de la asamblea, delante de la pizarra digital y en gran grupo.

- SESIÓN 2: Actividad 2. "Tocamos con instrumentos una canción india".

- Duración: Esta sesión tiene una duración de 60 minutos.

- Desarrollo:

Esta actividad se va a llevar a cabo en el aula de música, donde disponemos de gran variedad de instrumentos. Para su desarrollo, dividiremos a los alumnos en 3 equipos de 8 componentes cada uno, coincidiendo con los grupos de clase. Posteriormente, a cada grupo de alumnos se le dará un modelo de instrumento, repartiéndole tantos instrumentos como alumnos hay en el grupo. Antes de repartir a cada grupo los instrumentos, se los presentaremos y dejaremos que los toquen y experimenten con ellos de forma libre. A continuación, seguiremos los siguientes pasos para llevar a cabo la actividad:

○ Presentación de la canción.

- Tras escuchar la canción la primera vez, enseñaremos a los niños un ritmo concreto. Después, iremos dando instrucciones a los alumnos para indicarles en qué momento deberán intervenir y cuándo suena cada grupo de instrumentos.

\section{- Materiales:}

Los materiales que se van a utilizar para el desarrollo de esta actividad son el ordenador, los 
altavoces y los diferentes instrumentos, entre los que encontramos el triángulo, las maracas, la pandereta, los cascabeles y el tambor, entre otros.

- Organización:

Para el desarrollo de esta sesión, la clase estará dividida en 3 grupos de 8 componentes cada uno.

- SESIÓN 3: Actividad 3. "Elaboramos un palo de lluvia"

- Duración: Esta sesión tendrá una duración de 60 minutos aproximadamente.

- Desarrollo:

Antes de realizar la actividad principal, enseñaremos a los alumnos en la PDI diferentes danzas y canciones que realizan los indios con ayuda de los palos de lluvia. Posteriormente, les mostraremos imágenes de instrumentos musicales y cotidiáfonos y explicaremos qué son para que conozcan las diferencias que existen entre ellos. A continuación, les enseñaremos diferentes palos de lluvia caseros hechos con materiales que les resultan familiares.

Para el adecuado desarrollo de esta actividad, el cotidiáfono se realizará paso a paso, trabajando todos los alumnos a la vez, de manera, que hasta que un paso no esté completamente terminado ninguno de ellos pasará al siguiente. Es importante destacar, que los rollos de papel con las púas clavadas y el precinto, vendrán preparados de casa por los padres, para evitar repartir a los alumnos materiales peligrosos como son dichos objetos punzantes. A continuación, se pegará papel charol alrededor del rollo. Seguidamente, en el centro de cada equipo se pondrán gomets para que el alumnado los pegue alrededor para decorarlo. 1

\section{- Materiales:}

Los materiales para la elaboración de esta actividad serán la pizarra digital, un rollo de cartón alargado como podría ser el de papel de cocina, púas, arroz, gomets, papel charol de colores, precinto, pegamento de barra, tijeras...

\section{- Organización:}

Esta actividad se va a desarrollar de manera individual, ya que cada alumno fabricará su propio palo de lluvia.SESIÓN 4: Actividad 4. "Excursión al monte: Bailamos alrededor del fuego". 
- Duración: Esta sesión tendrá una duración de 60 minutos.

\section{- Desarrollo:}

Esta actividad consiste en hacer una salida al monte, el cual se encuentra situado a menos de 100 metros del colegio. Al llegar, cogeremos ramas y piedras que pondremos en el centro para simular una hoguera y nos sentaremos alrededor haciendo un círculo y pintaremos las caras de los niños y niñas como si fuesen indios. Alrededor de esta, haremos la canción de los gestos que aprendimos en la sesión 1. A continuación, enseñaremos a los niños una danza con la canción de “Ani Kuni”, trabajada en la sesión 2. Y finalmente, con los palos de lluvia realizados en la sesión anterior bailaremos alrededor libremente.

\section{- Materiales:}

Para la realización de esta actividad no se va a utilizar ningún tipo de material en concreto, únicamente el que nos proporcione el medio natural en el momento y nuestros palos de lluvia.

\section{- Organización:}

Esta actividad se va a desarrollar en gran grupo en el monte.

\section{METODOLOGÍA}

Teniendo en cuenta todo aquello que aparece en el currículo, entendemos este apartado como el conjunto de estrategias, procedimientos y todas aquellas acciones organizadas y planificadas por el profesorado de manera consciente y reflexiva, con la finalidad de posibilitar el aprendizaje del alumnado y el logro de los objetivos planteados.

Comobien manifiesta Veja “debemos proporcionar instrumentos y mecanismos de análisis que nos permitan mirar de frente a la realidad contemporánea, no para evadirnos del mundo sino para reconocernos en él como protagonistas y partícipes en el drama de nuestra época y para contribuir a afrontarlo". En este sentido consideramos que no hay que desterrar los libros de texto, pero sí usarlos como uno más, para ello hemos utilizado diferentes metodologías, estrategias y técnicas que nos ayudan a utilizar una variedad de recursos de manera consciente y adecuada, utilizando estrategias didácticas y actividades pedagógicas adecuadas, para que aprendan de manera transversal e interdisciplinar, es decir, los contenidos de cada ciencia deben relacionarse unos con otros para poder analizar la acción de los grupos sociales de un modo integrado, promoviendo aprendizajes integrales.

Como ya hemos dicho anteriormente los conceptos deberán adaptarse a los diferentes grados de comprensión del alumnado, para ello exteriorizamos sus conocimientos previos, organizado a través del 
dialogo, formulando preguntas para profundizar o ampliar contenidos.

Para conseguir todo esto, en nuestra unidad didáctica hemos decidido trabajar y enseñar el tema de los indios.

A continuación, mostramos las implicaciones metodológicas tomadas del plano legislativo. De acuerdo con el Decreto 254/2008, los métodos se basarán en la experiencia, actividad y juego dentro de un ambiente de seguridad, afecto y confianza, potenciando así la autoestima e integración social. En este sentido nos tomamos muy enserio el término juego, no establecemos una dicotomía entre juego y trabajo, si no que vamos a denominarlo a ambas como actividad. Además, proporcionaremos aspectos que promuevan la seguridad (como espacios protegidos de cualquier caída, goma eva en las esquinas, cajas delante de los radiadores...), el afecto (hablamos con cariño y afecto), la confianza (preparamos los espacios adaptados a su edad, tamaño y dificultades tanto psíquicas como físicas). Todas las experiencias se abordan desde un enfoque integrado y globalizador, orientando todos los momentos y actividades de manera ordenada.

La LOE determina que esta etapa debe contribuir al desarrollo físico, afectivo, social e intelectual. Pensamos que hay que individualizar también la enseñanza para abarcar estos ámbitos de desarrollo, por lo que tomamos diferentes medidas dependiendo del contexto, en este caso todas las actividades planteadas pueden realizarlas todos sin problemas, por lo que no suponen adaptaciones en ellas. Esta clase presenta una niña con un diagnóstico inicial de trastorno del espectro autista, para la cual realizaremos diferentes adaptaciones en las actividades que se desarrollarán en esta unidad didáctica, las cuales especificaremos en otro apartado.

Es importante destacar que se enseñan y aprenden las tres áreas del conocimiento del currículo de Educación Infantil. Y todo ello siguiendo la metodología expresada en el currículo.

Ante todo, proporcionamos aprendizajes relevantes, aprendizajes donde los alumnos se impliquen activamente en la tarea. Además, practicamos la socialización y se desarrolla la autonomía. Como se puede apreciar educamos desde y para la colectividad, casi todo se realiza en grupos, aunque hay momentos de trabajo individual, por lo que sistematizamos la cooperación. Organizamos el contexto escolar desde la temporalidad, estableciendo ritmos, rutinas y horario semanal.

Nuestra enseñanza se caracteriza por ser seria, reflexiva, informada, responsable y actualizada. Creo que estas singularidades han sido claramente expresadas, por lo que no vamos a mostrar ejemplos de estas. Nuestra principal técnica es la enseñanza a través de la música.

Además, es importante destacar que para la enseñanza de las diferentes canciones seguiremos siempre los mismos o similares pasos, dependiendo de lo que se quiera hacer y conseguir. Siendo estos los siguientes: 
1. Presentación del texto.

2. Presentación del tono melódico.

3. Acompañamiento.

4. Gestos.

5. Instrumentos.

6. Juego.

De las tres estrategias metodológicas de Quinquer, Dolors (2004) escogemos los métodos interactivos e individuales (con materiales de aprendizaje mediante las TIC). Damos prioridad a la cooperación, interacción y participación. Para favorecer su aprendizaje utilizaremos estrategias para procesar la información procedente de diferentes fuentes (fuentes primarias traídas por el docente y alumno de sus casas que serán utilizadas). Este proceso está organizado en fases según el tipo de tareas, estas son:

- Inicial: motivación, interrogantes, intercambio de ideas, propuestas.

- Desarrollo: obtención de nueva información, confrontación con nuevas ideas, es decir con la realidad.

- Síntesis: estructuración (reflexión y conclusión) comparación.

- Final: utilización de lo aprendido, consolidar más utilidad.

A modo de conclusión, creemos que superamos todos los modelos educativos que Trueba esboza en 1997, es decir tras el análisis de nuestra práctica docente vemos que nos encontramos en el modelo interactivo, con espacios descentralizados y organizados con intencionalidad, provocando situaciones que despiertan la curiosidad de nuestro alumnado. Somos unos catalizadores de sus intereses y colaboramos con el profesorado y familias para que todo pueda funcionar de manera correcta.

En definitiva, vemos que las estrategias de aprendizaje son: el diálogo, el debate, la creatividad, la exposición, el juego y el trabajo con fuentes primarias, lo que ayuda a generar nuevos conocimientos, motivando al niño a seguir aprendiendo.

\section{RECURSOS}

Para comenzar, decir que en el aula en la que nos encontramos y en la que hemos realizado esta interesante unidad didáctica sobre los indios, contamos con una niña con un diagnóstico inicial de trastorno del espectro autista, por lo que hemos tenido que realizar diversas adaptaciones para que pueda conseguir todos aquellos objetivos que queremos conseguir con el desarrollo de esta unidad didáctica.

En cuanto a los recursos materiales, el aula cuenta con mesas y sillas en los que los niños y niñas pueden trabajar cómodamente, ya que están adaptados a su altura.

Los materiales que hay en el aula se pueden clasificar en fungibles, que son aquellos que se gastan, 
como por ejemplo los lápices, rotuladores, temperas, pegamentos, folios, etc. Por otro lado, están los materiales no fungibles, es decir, que no se agotan, como por ejemplo las tijeras, punzones, pinceles, cajas donde guardar material, rodillos, moldes de plastilina... dentro de estos estarían los instrumentos musicales.

Otro recurso que hemos utilizado durante la unidad didáctica han sido la pizarra digital; para enseñarles todo de manera más atractiva a los niños pues una explicación de forma oral no es tan interesante como un video o cualquier otra visualización que podamos poner en la pizarra digital.

YouTube es otro recurso muy utilizado durante estas semanas, en el hemos encontrado todas las canciones y videos que les hemos enseñado a los niños y niñas para conseguir algunos de los objetivos propuestos para este proyecto.

Otros recursos que no han sido materiales, son los recursos humanos. Con esto nos referimos a las personas que nos han servido de gran ayuda para conseguir los objetivos y contenidos del proyecto. Además de todos los docentes que nos han ayudado a conseguir que la unidad didáctica saliera adelante. También podemos incluir a todos los alumnos y alumnas de infantil y a las familias que nos han facilitado la elaboración del palo de lluvia.

\section{MEDIDAS DE ATENCIÓN A LA DIVERSIDAD}

Para dar respuesta a las distintas necesidades de los alumnos y alumnas es necesario llevar a cabo unas medidas de atención a la diversidad. Por medidas se entienden aquellas actuaciones de índole tanto organizativa como curricular que se puedan llevar a cabo en el proceso de planificación o en el desarrollo de los procesos de enseñanza-aprendizaje.

Como ya hemos dicho anteriormente, el aula en la que se va a llevar a cabo esta Unidad Didáctica, existen diferentes grupos con ritmos de aprendizaje distintos, por lo que se pretenderá ayudar cuando sea necesario a los alumnos/as que lo requieran. Para ello, llevaremos a cabo una metodología que tendrá en cuenta estrategias como el aprendizaje cooperativo, el diseño de actividades graduables y actividades para atender los diferentes niveles de aprendizajes.

En cuanto a la niña con N.E.E. que presenta un trastorno generalizado del desarrollo, encontrándose dentro del espectro autista, se llevaran a cabo distintas medidas teniendo en cuenta las características que esta alumna presenta. Las sesiones de esta Unidad Didáctica se llevarán a cabo en gran grupo, con esto lo que se pretende es que la alumna se integre en el grupo clase y participe en las actividades intentando que esté lo más atenta posible y se aísle de lo que sucede lo menos posible.

La acción comunicativa de esta alumna es muy limitada, principalmente su forma de hablar es con ecolalias, repite involuntariamente una palabra o frase que acaba de pronunciar otra persona en su 
presencia, a modo de eco. Esto es bueno a la hora de que esta alumna se aprenda las letras de las canciones, ya que tiene una capacidad de memorización muy buena. Intentaremos fomentar esa acción comunicativa con el resto del grupo en las diferentes sesiones.

Finalmente, decir que esta alumna también presenta una pequeña sensibilidad auditiva, por lo que los ruidos de gran intensidad o la música con gran volumen hacen que se ponga nerviosa y quiera dejar de participar en la actividad, por lo que intentaremos adecuar el sonido para que este no sea demasiado elevado con el fin de evitar que esta alumna se sienta incomoda realizándola.

\section{INTERDISCIPLINARIEDAD DE ÁREAS Y EDUCACIÓN EN VALORES}

Como ya hemos dicho al inicio, esta Unidad Didáctica surge del interés de los alumnos y alumnas por el tema de los indios de América, y paralelamente a esta Unidad de música, se va a utilizar este tema para trabajar todas las áreas del currículo. Además, al partir de un eje temático en esta Unidad Didáctica se trabajan distintos aprendizajes a parte de la música, por lo que hablamos de una Unidad Interdisciplinar, en la que están presentes otras enseñanzas que se imparten en la Educación Infantil.

Finalmente, decir que, con esta unidad didáctica, también se trabaja la educación en valores, con esto lo que vamos a pretender con todas las sesiones se trabajen valores como el respeto, la tolerancia, la libertad de expresión y el compañerismo. La finalidad de esto es para que exista una muy buena cohesión del grupo-clase, y queremos destacar el cariño y el respeto con el que tratan a la niña con N.E.E., todos los niños/as del aula las ayudan e intentan integrarla de forma activa en todas las actividades.

\section{EVALUACIÓN}

Para llevar a cabo la evaluación de esta unidad didáctica referida al ámbito musical vamos a utilizar una escala de estimación con tres valores que serán los siguientes: conseguido, en proceso y no conseguido. Para cumplimentar dicho instrumento de evaluación, lo haremos a través de la observación directa y sistemática de todo el grupo de alumnos y alumnas. Esto es, durante la realización de las actividades o sesiones podremos completar las tablas de los diferentes niños situando una marca en forma de cruz en el interior del cuadro correspondiente según el momento en el cada uno se encuentre en los diferentes ítems de evaluación que hayamos establecido. Además, utilizaremos un diario para ir tomando nota de informaciones que consideremos relevantes para que sea más fácil rellenarla y para tenerlas en cuenta posteriormente en otras unidades didácticas y en otras materias.

A continuación, plasmaremos la tabla modelo que usaremos en la que aparecen los criterios de evaluación que hemos considerado más adecuados en relación con esta unidad didáctica y con el currículo del segundo ciclo de educación infantil: 
Tabla 4. Evaluación del alumnado. Fuente: elaboración propia.

Nombre del alumno:

\begin{tabular}{|c|c|c|c|c|}
\hline & Conseguido & $\begin{array}{l}\text { En } \\
\text { proceso }\end{array}$ & $\begin{array}{l}\text { No } \\
\text { conseguido }\end{array}$ & Observaciones \\
\hline \multicolumn{5}{|l|}{$\begin{array}{l}\text { Ha observado y explorado el entorno } \\
\text { natural. }\end{array}$} \\
\hline \multicolumn{5}{|l|}{$\begin{array}{l}\text { Relaciona su entorno cercano con la } \\
\text { temática tratada. }\end{array}$} \\
\hline \multicolumn{5}{|l|}{$\begin{array}{l}\text { Conoce los indios de América, su } \\
\text { cultura, hábitos y costumbres. }\end{array}$} \\
\hline \multicolumn{5}{|l|}{$\begin{array}{l}\text { Identifica los } \quad \text { instrumentos } \\
\text { característicos de los indios y su uso. }\end{array}$} \\
\hline \multicolumn{5}{|l|}{$\begin{array}{l}\text { Interpreta canciones propias de los } \\
\text { indios americanos. }\end{array}$} \\
\hline \multicolumn{5}{|l|}{$\begin{array}{l}\text { Es capaz de usar la expresión } \\
\text { corporal para } \\
\text { canciones, cantando y bailando. }\end{array}$} \\
\hline \multicolumn{5}{|l|}{$\begin{array}{l}\text { Puede seguir ritmos indicados por la } \\
\text { profesora. }\end{array}$} \\
\hline \multicolumn{5}{|l|}{$\begin{array}{l}\text { Desarrolla habilidades motrices de equilibrio } \\
y \quad \text { coordinación } \\
\text { para } \\
\text { realizar una danza. }\end{array}$} \\
\hline \multicolumn{5}{|l|}{$\begin{array}{l}\text { Conoce las cualidades sonoras de } \\
\text { su voz. }\end{array}$} \\
\hline \multicolumn{5}{|l|}{$\begin{array}{l}\text { Utiliza adecuadamente la percusión } \\
\text { corporal. }\end{array}$} \\
\hline \multicolumn{5}{|l|}{$\begin{array}{l}\text { Desarrolla habilidades motrices de } \\
\text { carácter fino. }\end{array}$} \\
\hline \multicolumn{5}{|l|}{$\begin{array}{l}\text { Confecciona instrumentos típicos de los indios } \\
\text { mediante técnicas } \\
\text { plásticas conocidas. }\end{array}$} \\
\hline $\begin{array}{l}\text { Diferencia entre } \\
\text { musicales y cotidiáfonos. }\end{array}$ & & & & \\
\hline
\end{tabular}

\section{EVALUACIÓN DE LA PRÁCTICA DOCENTE}

Además de la evaluación que realizaremos a los alumnos y alumnas para comprobar si han adquirido los conocimientos y habilidades que se pretendían con esta unidad didáctica también nos evaluaremos nosotros como maestros, incluyendo aquí el proyecto en sí, su utilidad y nuestra actuación docente, además de añadir alguna propuesta de mejora si lo consideramos conveniente. Para ello y a modo de ejemplo, podríamos rellenar una tabla como la siguiente:

\section{PROFESORA:}

Tabla 5. Evaluación de la práctica docente. Fuente: elaboración propia.

\section{ASPECTOS A TENER EN CUENTA}

Se trabaja de forma global en la unidad didáctica.
VALORACIÓN

PROPUESTA DE MEJORA 
Las actividades son motivadoras e interesantes.

El orden de las sesiones es adecuado.

Se ha tenido en cuenta los intereses y motivaciones de los alumnos.

Los alumnos han descubierto toda la información que pretendíamos sobre la unidad didáctica.

$\begin{array}{lrrr}\text { Ha sido fácil para } & \text { los } & \text { alumnos } \\ \text { conseguir } & \text { los } & \text { objetivos } & \text { que } \\ \text { proponíamos. } & & & \end{array}$

El rendimiento de la clase ha sido positivo.

Los criterios de evaluación son
concretos.

Tienen relación los contenidos con los objetivos del proyecto.

Estoy satisfecho con mi tarea como docente. 


\section{REFERENCIAS BIBLIOGRÁFICAS}

CARM (2008). Decreto número 254/2008, de 1 de agosto (BORM n. ${ }^{\circ} 182$, de 06/08/2008), por el que se establece el currículo del Segundo Ciclo de Educación Infantil en la Comunidad Autónoma de la Región de Murcia.

YouTube. (2008). Recuperado de: https://www.youtube.com/watch?v=0VeG6B3jtKI

YouTube. (2012) Recuperado de: https://www.youtube.com/watch?v=TVjq61LV9ts 